Technische

Universität

Berlin

Chuang Shi, Shiwei Guo, Shengfeng Gu, Xinhao Yang, Xiaopeng Gong, Zhiguo Deng, Maorong Ge, Harald Schuh

\title{
Multi-GNSS satellite clock estimation constrained with oscillator noise model in the existence of data discontinuity
}

Open Access via institiutional repository of Technische Universität Berlin

Document type

Journal article | Accepted version

(i. e. final author-created version that incorporates referee comments and is the version accepted for publication; also known as: Authors Accepted Manuscript (AAM), Final Draft, Postprint)

This version is available at

https://doi.org/10.14279/depositonce-11981

\section{Citation details}

Shi, C., Guo, S., Gu, S., Yang, X., Gong, X., Deng, Z., Ge, M., \& Schuh, H. (2018). Multi-GNSS satellite clock estimation constrained with oscillator noise model in the existence of data discontinuity. Journal of Geodesy, 93(4), 515-528. https://doi.org/10.1007/s00190-018-1178-3

This is a post-peer-review, pre-copyedit version of an article published in Journal of Geodesy. The final authenticated version is available online at: https://doi.org/10.1007/s00190-018-1178-3.

Terms of use

This work is protected by copyright and/or related rights. You are free to use this work in any way that is permitted by the copyright and related rights legislation that applies to your use. For other uses you need to obtain permission from the rights-holder(s). 
Journal of Geodesy manuscript No.

(will be inserted by the editor)

\title{
Multi-GNSS satellite clock estimation constrained with oscillator noise model in the existence of data discontinuity
}

Chuang Shi · Shiwei Guo · Shengfeng Gu •

Xinhao Yang · Xiaopeng Gong · Zhiguo

Deng · Maorong Ge · Harald Schuh

Received: date / Revised version: date

\begin{abstract}
During the past years, Real-time Precise Point Positioning (PPP) has been proven to be an efficient tool in the applications of navigation, precise orbit determination of LEO as well as earthquake and tsunami early warning, etc. One of the most crucial issues of these applications is the high precision real-time GNSS satellite clock. Though the performance and character of the GNSS onboard atomic frequency standard have been widely studied, the white noise model is still the most popular hypothesis that employed in the real-time GNSS satellite clock estimation. However, concerning the real-time applications, significant data discontinuity may arise either due to the fact that only regional stations involved, or the failure in the stations, satellites and network connections. These data discontinuity would result in an arbitrary clock jump between adjacent arcs when the clock offsets are modelled as white noise. In addition, it is also expected that the detection and identification of outliers would be benefited from the constrains of the satellite oscillator noise model. Thus in this contribution, based on the statistic analysis of almost two years multi-GNSS precise clock products, we developed the oscillator noise model for the satellites of GPS, GLONASS, BDS and Galileo according to the oscillator type as well as the block type. Then, the efficiency of this oscillator noise model in multi-GNSS satellite clock estimation is demonstrated with two months data for both regional and global networks in simultaneous realtime mode. For the regional network, the results suggest that compared with the traditional solution based on white noise model, the improvement is $44.4 \%$ and

Chuang Shi · Shiwei Guo · Shengfeng Gu · Xinhao Yang · Xiaopeng Gong

GNSS Research Center, Wuhan University, 129 Luoyu Road, Wuhan 430079, China

Chuang Shi · Shiwei Guo

School of Electronic and Information Engineering, Beihang University, 37 Xueyuan Road, Beijing 100083, China

Shengfeng Gu $\cdot$ Zhiguo Deng $\cdot$ Maorong Ge $\cdot$ Harald Schuh

Helmholtz Centre Potsdam, German Research Centre for Geosciences (GFZ), Telegrafenberg, 14473 Potsdam, Germany

Harald Schuh

Technische Universität Berlin, 10623 Berlin, Germany

*corresponding author: gsf@whu.edu.cn
\end{abstract}


$12.1 \%$ on average for STD and RMS, respectively, and the improvement is mainly attributed to the efficiency of the oscillator noise model during the convergence period and the gross error resistance. Concerning the global experiment, since the stations guarantee the continuous tracking of the satellites with redundant observable, the improvement is not as evident as that of regional experiment for GPS, GLONASS and BDS. While, the STD of Galileo clock improves from 0.28ns to $0.19 n s$ due to that, the satellites E14 and E18 still suffer significant data discontinuity during our experimental period.

Keywords Clock model, Data discontinuity, Multi-GNSS, Real-time

\section{Introduction}

The potential of Global Navigation Satellite Systems (GNSS) as an efficient tool in providing precise positioning has been widely recognized nowadays. Specifically, the so-called Precise Point Positioning (PPP) is one of the most promising techniques because of its cost-effectiveness, global coverage and high accuracy [Zumberge et al. (1997), Kouba and Héroux. (2001), Bisnath and Gao (2008)]. Moreover, in order to meet the growing requirements of real-time PPP applications, e.g. navigation, precise orbit determination of LEO, earthquake and tsunami early warning and GNSS-based weather forecasting, etc, the International GNSS Service (IGS) has issued a call for participation in IGS Real-time Pilot Project (IGS-RTPP) since 2007, and provided the precise orbit and clock correction for GPS and GLONASS officially in April 2013 [Weber et al. (2007), Shi et al. (2013), Zhang et al. (2015)].

Accuracy of orbit and clock products are fundamental to guaranty the performance of PPP. The predicted part of orbit provided by IGS ultra-rapid ephemeris in real-time has precision of centimeter level, which is suitable for PPP applications. The predicted clock of ultra-rapid product, however, deviates quickly due to the frequency variation originated from a variety of effects, e.g., ambient temperature, supply voltages, magnetic field, etc. Thus, the real-time estimation of satellite clocks is one of the key objectives of high precision GNSS service.

Obviously, a thorough understanding of the behavior of Atomic Frequency Standard (AFS) equipped on GNSS is useful for an optimal estimation of satellite clocks. And attribute to the successful deployment and wide-spread of GNSS, the stability of these onboard atomic oscillator has been analyzed in details since the early 1990s within the frequency transfer community [Petit and Thomas (1996)]. Based on the two-sample variance, referred as Allan variance, a number of efforts has been made to derive the noise spectral density of GPS Cesium oscillator, which is essential to characterize the frequency fluctuations of atomic clocks [Swift and Hermann (1988), Wübbena (1988)]. Along with the GPS program progress, increasing satellites are equipped with Rubidium clocks. As pointed out by [Hutsell (1995)], the Rubidium oscillator exhibits significant frequency drift (aging) and aging noise characteristics, which may leave the Allan variance analysis divergent. Thus, the three-sample variance, i.e., Hadamard variance, which removes the linear frequency aging automatically, presented an appropriate tool for characterizing these AFS noise [Hutsell (1995)]. By implementation of these two techniques, a wide range of valuable literatures has been published in parallel to the upgrading of the GPS satellites, based on a much wider 
scope of clock observables with an improved quality from IGS [Wright (2007), Senior et al. (2008), Huang et al. (2013A)]. More recently, along with the modernization of GPS and GLONASS, and the newly deployed BDS and Galileo, the oscillator stability of the new generation GNSS has received increasing interests again [Montenbruck et al. (2012), Steigenberger et al. (2013), Wang et al. (2017)]. It is concluded that the typical Allan variance of daily frequency stability is between $10^{-14}$ and $10^{-13}$ for the GNSS onboard oscillators [Steigenberger et al. (2013), Huang et al. (2013A)].

It is noted that most of the above studies are focused on the performance of onboard oscillator and frequency transfer based on the GNSS common-view technique. Concerning the satellite clock determination, however, the white noise model is still the most popular hypothesis that is underlining the parameter estimation. On the one hand, taking the high-resolution requirement of real-time applications into consideration, the corresponding clock estimation turns out to be too time-consuming to support PPP with a sampling rate up to $1 \mathrm{~Hz}$. It is the reason why great efforts are underway in direction of an efficient satellite clock solution within the GNSS community. Among which, [Bock et al. (2009A)] proposed to perform low rate, i.e., 5 min, clock estimation and receiver clock synchronization using code measurements, and then to densify them with the clock increments derived from the epoch-differenced phase measurements. Similarly, the clock estimation model based on the un-differenced pseudo-range and epoch-differenced carrier-phase was also suggested by [Ge et al. (2011)], but in a real-time fashion. Obviously, the computation can be accelerated in the procedure of epoch-difference as the elimination of ambiguities. In contrast, the employment of the AFS noise model implies an extra computational burden in the satellite clock solution, since the oscillator noise model typically involves the simultaneous estimation of clock error, frequency offset, and aging. On the other hand, as evidenced by a wide range of publications, the performance of the clock corrections generated with the white noise model seems to work fairly well with an overall statistics of about $0.1 \mathrm{~ns}$, which is sufficient for the centimeter level positioning with a stand-alone receiver [Bock et al. (2009B), Laurichesse et al. (2013), Zhang et al. (2015)].

However, as far as the real-time PPP applications are concerned, the problem is more complicated. Firstly, the assumption that the satellites can be continuously tracked relies on the distribution of the stations involved as well as the network condition between the stations and the real-time processing facility. However, there are numerous cases that only regional station's observables are available [Shi et al. (2017)]. In addition, a significant amount of data might be lost or arrive too late for processing due to network problems [Hauschild and Montenbruck (2009)]. This data discontinuity will result in an arbitrary clock jump between adjacent arcs when the clock offsets are modelled as white noise parameters, and consequently cause a degradation of the PPP performance. Secondly, the detection and identification of outlier satellites is more challenging under the practical environment, and it is expected that the filter robustness may benefit from the predicted satellite clock offsets based on the oscillator noise model. Last but not least, though the effect is potentially not harmful to positioning, it should be mentioned that a more stable satellite clock value constrained with an a priori oscillator noise model is always encouraged in the applications of GNSS based timing and synchronization. Unfortunately, to the best of our knowledge, only [Hauschild and Montenbruck (2009), Hauschild (2010)] have absorbed the oscilla- 
tor noise model in real-time GPS clock estimation, and with an identical process noise setting for all the satellites. In addition, they have also failed to fully excavate the potential of oscillator noise model constrains in the resistance to the data discontinuity for clock estimation.

It is generally acknowledged that the satellite clock behavior depends on AFS oscillator type as well as block type [Senior et al. (2008)]. In addition, as evidenced by [Lou et al. (2015)], compared with the GPS only solution, better performance of PPP would be achieved by employing the multi-GNSS constellations. Furthermore, an efficient solution for real-time GNSS network processing has been promoted by [Gong et al. (2017)], in which the epoch multi-GNSS clock generation based on undifferenced pseudo-range and carrier-phase is around $0.3 \mathrm{~s}$ with over 80 globally distributed stations.

These deficiencies of the previous studies as well as the achievements in realtime multi-GNSS data processing recently as discussed above are the main drivers of this contribution, in which, we have demonstrated the efficiency of the AFS model in real-time multi-GNSS clock estimation in case of discontinued satellite tracking. This paper is organized as follows: after the introduction of the basic multi-GNSS data processing model with the emphasize on the oscillator model; the oscillator noise model for GPS, GLONASS, BDS and Galileo are studied and promoted; with the oscillator model, both regional and global satellite clock estimation are performed and analyzed in simulate real-time mode.

\section{Method}

\subsection{Multi-GNSS Data Processing Model}

In this paper, the satellite system identifiers ( $G R C E$ ) as suggested in RINEX 3.02 format are used to denote GPS, GLONASS, BDS and Galileo, respectively. The linear combination known as ionosphere-free (IF) that is typically involved in the satellite clock estimation for a specific GNSS system sys (sys $\in(G R C E)$ ) is expressed as

$$
\left.\begin{array}{rl}
P_{r}^{s} & =p_{r}^{s}-\rho_{r}^{s} \\
& =t_{r, s y s}-t^{s}+\alpha_{r}^{s} \cdot T_{r}^{z}+e_{s y s} \cdot b_{r}^{k} \\
\Phi_{r}^{s} & =\phi_{r}^{s}-\rho_{r}^{s} \\
& =t_{r, s y s}-t^{s}+\alpha_{r}^{s} \cdot T_{r}^{z}+e_{s y s} \cdot b_{r}^{k}-\lambda \cdot N_{r}^{s}+\varepsilon_{\phi}
\end{array}\right\}
$$

in which, $P_{r}^{s}, \Phi_{r}^{s}$ are observation minus calculated (OMC) for pseudo-range and carrier-phase from receiver $r(r=1, \cdots, i)$ to satellite $s(s=1, \cdots, j)$ in length units, respectively; where $i$ is the number of receivers involved, and $j$ is the number of satellites being tracked; $p_{r}^{s}, \phi_{r}^{s}$ are pseudo-range and carrier-phase observable; $\rho$ is the geometric distance, while the antenna phase center, tidal displacements, relativity, phase windup corrections should be applied to $p, \phi$ before $\rho$ becomes unassociated with the frequency as well as the system sys; $t_{r, s y s}$ is the clock offset corresponding to receiver $r$ for the system sys; $t^{s}$ denotes the clock offset of satellite $s ; T_{r}^{z}$ is the zenith tropospheric delay that can be converted to slant with the mapping function $\alpha ; b_{r}^{k}$ is the receiver code bias with the corresponding coefficient $e_{s y s} ; N$ is the float ambiguity in cycle, together with the corresponding wavelength $\lambda$. 
Note that the satellite code bias is not presented since the corresponding DCB products of IGS are held fixed in this study. Regarding the receiver code bias term $b_{r}^{k}$, the coefficient $e_{s y s}$ is related to the satellite system [Lou et al. (2015), Liu et al. (2017)]

$$
e_{\text {sys }}=\left\{\begin{array}{l}
0, C D M A(G, C, E) \\
1, F D M A(R)
\end{array}\right.
$$

since for the CDMA based systems, the receiver code bias is identical for different satellite, and thus it is absorbed in the receiver clock $t_{r, s y s}$. While for the GLONASS with FDMA signal, the receiver code bias $b_{r}^{k}$ is satellite frequency number $k$ related, in which $k=(-7-4-3 \cdots 0 \cdots 6)$ (http://www.glonasscenter.ru).

Regarding the dynamic model for these parameters, it is typically assumed that the clocks for receiver and satellite are estimated as white noise, $\boldsymbol{b}_{\boldsymbol{r}}$ and $\boldsymbol{N}_{\boldsymbol{r}}$ are constant parameters, while the troposphere is regarded as random walk which satisfies

$$
T_{r, n+1}^{z}=T_{r, n}^{z}+\omega_{t^{z}, n}
$$

in which $\omega_{t^{z}, n}$ is a random process with

$$
\left.\begin{array}{ll}
E\left(\omega_{t}, n\right. & =0 \\
D\left(\omega_{t^{z}, m}, \omega_{t^{z}, n}\right) & =Q_{t^{z}} \cdot \delta_{m, n}
\end{array}\right\}
$$

and $\delta_{m, n}$ is the Kronecker delta function, which is zero except when $m=n$, then $\delta_{n, n}=1$ and $Q_{t}$ is set as $15 \mathrm{~mm} / \sqrt{h}$, typically.

\subsection{Oscillator Noise Model}

Since every oscillator, atomic or crystal, deviates from the ideal or "true" time in an abstract sense except as we may choose to define it [Allan (1987)]. The satellite clock bias $t^{s}(n)$ of a given epoch $n$ emerged from the offset between the instantaneous reading of the clock $t_{r}^{s}(n)$ and the reference clock $t^{0}(n)$, can be expressed as

$$
\begin{aligned}
t^{s}(n) & =t_{r}^{s}(n)-t^{0}(n) \\
& =\left(t_{0}^{s}+\int_{0}^{n} \frac{f^{s}(t)}{f^{0}} d t\right)-\left(t_{0}+\int_{0}^{n} \frac{f^{0}}{f^{0}} d t\right) \\
& =\left(t_{0}^{s}-t_{0}\right)+\int_{0}^{n} \frac{f^{s}(t)-f^{0}}{f^{0}} d t \\
& =a_{0}+\int_{0}^{n} y(t) d t
\end{aligned}
$$

where $t_{0}^{s}$ and $t_{0}$ is the clock reading of the oscillator and the "true" clock of a selected reference epoch; $f^{s}(t)$ is the instantaneous frequency of the oscillator; $f^{0}$ is the nominal frequency; $a_{0}$ is the clock bias at the reference epoch; $y(t)$ is by definition the instantaneous frequency deviation.

Though the satellite clock term $t^{s}$ is typically estimated as a white noise parameter, the sophisticated clock model is still a requirement for a more robust solution of real-time GNSS applications. Generally, the instantaneous oscillator frequency is assumed to be of the form

$$
f^{s}(t)=f^{0}+d f+\sum_{m=1}^{N-1} \frac{f^{(m)}}{m !} \cdot t^{m}+\varepsilon(t)
$$


in which, $d f$ is the mean frequency bias from the nominal frequency $f_{0} ; f^{(m)}$ is the $m$ th-order frequency drift, that dominates the long-term instability; $\varepsilon(t)$ is the random frequency errors, that describe the short-term instability [Lindsey (1976)].

Thus from Eq. (5) and Eq. (6), the instantaneous frequency deviation reads

$$
y(t)=\frac{d f}{f^{0}}+\sum_{m=1}^{N-1} \frac{f^{(m)}}{f^{0} \cdot m !} \cdot t^{m}+\frac{\varepsilon(t)}{f^{0}}
$$

Substituting Eq. (7) into Eq. (5), we derive the general model of clock error

$$
t^{s}(n)=a_{0}+\frac{d f}{f^{0}} \cdot n+\sum_{m=2}^{N} \frac{f^{(m-1)}}{f^{0} \cdot m !} \cdot n^{m}+\int_{0}^{n} \frac{\varepsilon(t)}{f^{0}} d t
$$

The rearranging of terms and a new notation yields the clock error

$$
t^{s}(n)=a_{0}+a_{1} \cdot n+\frac{a_{2}}{2} \cdot n^{2}+\sum_{m=3}^{N} \frac{f^{(m-1)}}{f^{0} \cdot m !} \cdot n^{m}+r(n)
$$

where $a_{0}$ is defined as Eq. (5); $a_{1}$ is the frequency bias; $a_{2}$ is the frequency drift; $r(t)$ is the integrated random fractional frequency error [Wübbena (1988)].

By selection the proper degree, i.e., $N$, in the model (8) and (9), different dimensional algorithms may be derived. As demonstrated by [Shmaliy et al. (1999)], three-dimension ( $\left.a_{0} a_{1} a_{2}\right)$ is the most efficient for the Rubidium oscillator, while the quadratic term $a_{2}$ brings less good results for the Cesium-beam standards [Allan (1987)], i.e.,

$$
\begin{aligned}
& t^{s}(n)=c^{\boldsymbol{T}} \cdot \boldsymbol{a}
\end{aligned}
$$

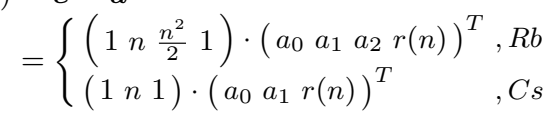

In addition, the dynamic equation of a satellite clock is written as

$$
a_{n+1}=\Phi_{a}(\tau) \cdot a_{n}+\omega_{a}
$$

with $\tau$ denotes the time interval of epoch $n$ and $n+1$, and

$$
\begin{aligned}
& \Phi_{a}(\tau)=\left\{\begin{array}{l}
\left(\begin{array}{llll}
1 & \tau & \frac{\tau^{2}}{2} & 0 \\
0 & 1 & \tau & 0 \\
0 & 0 & 1 & 0 \\
0 & 0 & 0 & 1
\end{array}\right), R b \\
\left(\begin{array}{lll}
1 & \tau & 0 \\
0 & 1 & 0 \\
0 & 0 & 1
\end{array}\right), C s
\end{array}\right. \\
& \boldsymbol{\omega}_{\boldsymbol{a}}=\left\{\begin{array}{lll}
\left(\begin{array}{llll}
0 & 0 & 0 & \omega_{r}
\end{array}\right), & R b \\
\left(\begin{array}{llll}
0 & 0 & \omega_{r}
\end{array}\right) & , C s
\end{array}\right.
\end{aligned}
$$




\section{Satellite clock}

\subsection{Temporal Statistics}

In section 2 , both the observable model and the dynamic model for multi-GNSS satellite clock estimation have been presented. However, the temporal statistical property of the satellite clock terms $\boldsymbol{a}$ is still under developed. Thus, to get the quantitative measure of the correlation for these terms, the repeatability of $\left(\begin{array}{lll}a_{0} & a_{1} & a_{2}\end{array}\right)$ and the variograms of $r$ are derived on the sub-daily regime in this section.

Since May. 3rd, 2015, GFZ begins to provide satellite clocks for $G, R, C, E$ with a resolution of up to $30 \mathrm{sec}$ which can be obtained from

http://doi.org/10.5880/GFZ.1.1.2017.002. Thus, near 2 years final precise multiGNSS clock products from May 3rd, 2015 to Feb. 25th, 2017 were collected in this statistical study based on model (10). It is noted that before this study, preprocessing should be carried out to get rid of the difficulties of large data gaps, outliers as well as the jump discontinuity across the processing boundary [Huang et al. (2012)].

The repeatability of $\left(\begin{array}{lll}a_{0} & a_{1} & a_{2}\end{array}\right)$ is obtained in terms of STD by the comparison of the 12-hour prediction and the fitted value, while the variograms of $r$ are obtained based on a finite sample with a robust method suggested by [Cressie and Hawkins (1980)] as:

$$
2 \hat{\gamma}(h) \equiv\left\{\frac{1}{|N(h)|} \sum_{N(h)}\left|r\left(s_{i}\right)-r\left(s_{j}\right)\right|^{1 / 2}\right\}^{4} /\left(0.457+\frac{0.494}{|N(h)|}\right)
$$

where $2 \hat{\gamma}(h)$ is the estimate of variogram; $r(s)$ is the satellite clock fitting residual of model (10) for epoch $s$; the sum is over $N(h) \equiv\left\{(i, j): s_{i}-s_{j}=h\right\}$ and $|N(h)|$ is the number of distinct elements of $N(h)$. From definition, the variogram $2 \gamma(h)$ relates to the covariance by the following equations

$$
2 \gamma\left(s_{i}, s_{j}\right)=2\left(C(0)-C\left(s_{i}-s_{j}\right)\right)
$$

with $C(\cdot)$ the covariogram which is defined by covariance directly

$$
C\left(s_{i}-s_{j}\right)=\operatorname{cov}\left(r\left(s_{i}\right), r\left(s_{j}\right)\right)
$$

Thus, the quantity variogram describes the temporal behavior of $r$ exactly.

In the following paragraph, we present the results from our experiment directly. Shown in Tab. 1 to Tab. 4 are the optimal dimension and the STD of $\left(\begin{array}{lll}a_{0} & a_{1} & a_{2}\end{array}\right)$ for $G, R, C, E$, respectively, and the oscillator informations are also presented. It should be noted, that the unit for $a_{1}$ and $a_{2}$ is $n s / h$ and $n s / h^{2}$, respectively.

Consistent with model (10), the optimal dimension for Rubidium and Cesium oscillator is 3 and 2 , respectively regardless the satellite constellation involved. The best prediction precision for $\left(\begin{array}{lll}a_{0} & a_{1} & a_{2}\end{array}\right)$ can be found in GPS, which is around $0.5 n s, 0.06 n s / h$ and $0.003 n s / h^{2}$, respectively, while, the repeatability decreases to $0.6 n s$ and $0.09 n s / h$ for GLONASS satellites. Concerning Galileo satellites, the performance of rubidium and hydrogen oscillator are roughly the same with a precision of $0.96 n s, 0.09 n s / h$ and $0.006 n s / h^{2}$ on average. Though the oscillator 
information of BDS is not available publicly, the 3-dimension algorithm is suggested for all its satellites from our experiment, and the statistics also suggests that the terms $\left(\begin{array}{lll}a_{0} & a_{1} & a_{2}\end{array}\right)$ of BDS is less stable compared with that of $\mathrm{G}, \mathrm{R}$ and E. Moreover it seems that the behavior of $\mathrm{C} 06$ is abnormal with a much larger STD value.

Table 1 Optimal algorithm dimension and the STD for $a_{0}, a_{1}$ and $a_{2}$ of GPS

\begin{tabular}{|c|c|c|c|c|c|c|}
\hline \multirow{2}{*}{ Sat. } & \multirow{2}{*}{ BLOCK } & \multirow{2}{*}{ Osc. } & \multirow{2}{*}{ Dim. } & \multicolumn{3}{|c|}{$12 \mathrm{~h}$ prediction STD } \\
\hline & & & & $\begin{array}{l}a_{0} \\
n s\end{array}$ & $\begin{array}{c}\boldsymbol{a}_{\mathbf{1}} \\
n s / h\end{array}$ & $\begin{array}{c}\boldsymbol{a}_{\mathbf{2}} \\
n s / h^{2}\end{array}$ \\
\hline G01 & IIF & $\mathrm{Rb}$ & 3 & 0.516 & 0.047 & 0.002 \\
\hline G02 & IIR & $\mathrm{Rb}$ & 3 & 0.378 & 0.041 & 0.004 \\
\hline G03 & IIF & $\mathrm{Rb}$ & 3 & 0.449 & 0.043 & 0.002 \\
\hline G05 & IIR-M & $\mathrm{Rb}$ & 3 & 0.757 & 0.069 & 0.004 \\
\hline G06 & IIF & $\mathrm{Rb}$ & 3 & 0.360 & 0.033 & 0.002 \\
\hline G07 & IIR-M & $\mathrm{Rb}$ & 3 & 0.463 & 0.047 & 0.004 \\
\hline G08 & IIF & Cs & 2 & 0.455 & 0.114 & \\
\hline G09 & IIF & $\mathrm{Rb}$ & 3 & 0.404 & 0.040 & 0.002 \\
\hline G10 & IIF & $\mathrm{Rb}$ & 3 & 0.515 & 0.047 & 0.002 \\
\hline G11 & IIR & $\mathrm{Rb}$ & 3 & 0.938 & 0.088 & 0.004 \\
\hline G12 & IIR-M & $\mathrm{Rb}$ & 3 & 0.801 & 0.068 & 0.003 \\
\hline G13 & IIR & $\mathrm{Rb}$ & 3 & 0.547 & 0.054 & 0.004 \\
\hline G14 & IIR & $\mathrm{Rb}$ & 3 & 0.369 & 0.040 & 0.004 \\
\hline G15 & IIR-M & $\mathrm{Rb}$ & 3 & 0.435 & 0.040 & 0.002 \\
\hline G16 & IIR & $\mathrm{Rb}$ & 3 & 0.712 & 0.063 & 0.003 \\
\hline G17 & IIR-M & $\mathrm{Rb}$ & 3 & 0.673 & 0.060 & 0.004 \\
\hline G18 & IIR & $\mathrm{Rb}$ & 3 & 0.630 & 0.054 & 0.003 \\
\hline G19 & IIR & $\mathrm{Rb}$ & 3 & 0.368 & 0.039 & 0.003 \\
\hline G20 & IIR & $\mathrm{Rb}$ & 3 & 0.390 & 0.041 & 0.003 \\
\hline G21 & IIR & $\mathrm{Rb}$ & 3 & 0.569 & 0.054 & 0.003 \\
\hline G22 & IIR & $\mathrm{Rb}$ & 3 & 0.465 & 0.046 & 0.003 \\
\hline G23 & IIR & $\mathrm{Rb}$ & 3 & 0.320 & 0.035 & 0.003 \\
\hline G24 & IIF & Cs & 2 & 0.631 & 0.158 & \\
\hline G25 & IIF & $\mathrm{Rb}$ & 3 & 0.532 & 0.050 & 0.002 \\
\hline G26 & IIF & $\mathrm{Rb}$ & 3 & 0.683 & 0.045 & 0.002 \\
\hline G27 & IIF & $\mathrm{Rb}$ & 3 & 0.557 & 0.051 & 0.002 \\
\hline G28 & IIR & $\mathrm{Rb}$ & 3 & 0.838 & 0.095 & 0.009 \\
\hline G29 & IIR-M & $\mathrm{Rb}$ & 3 & 0.590 & 0.056 & 0.003 \\
\hline G30 & IIF & $\mathrm{Rb}$ & 3 & 0.527 & 0.039 & 0.002 \\
\hline G31 & IIR-M & $\mathrm{Rb}$ & 3 & 0.591 & 0.055 & 0.004 \\
\hline G32 & IIF & $\mathrm{Rb}$ & 3 & 0.494 & 0.037 & 0.002 \\
\hline \multicolumn{4}{|c|}{ Ave. } & 0.547 & 0.056 & 0.003 \\
\hline
\end{tabular}

In addition, Fig. 1 to Fig. 4 present the quantitative measure of the temporal correlation of $r$ for each satellite. The dashed line denotes the experimental variogram computed from the fitting residual. Overall, the variogram increases along with the distance: it approaches to zero as $h$ approaches to zero and becomes a constant as $h$ increases up to several hours. The faster increase and larger value of variogram implies that the temporal behavior of residual $r$ is less stable. For GPS satellites, it is observed that the behavior of the random fractional frequency error $r$ depends on not only the oscillator type, but also the block type, and the BLOCK IIF satellite equipped with rubidium oscillator gives the best performance. While, 
Table 2 Optimal algorithm dimension and the STD for $a_{0}$ and $a_{1}$ of GLONASS

\begin{tabular}{|c|c|c|c|c|}
\hline \multirow{2}{*}{ Sat. } & \multirow{2}{*}{ Osc. } & \multirow{2}{*}{ Dim. } & \multicolumn{2}{|c|}{$12 \mathrm{~h}$ prediction STD } \\
\hline & & & $\begin{array}{l}a_{0} \\
n s\end{array}$ & $\begin{array}{c}\boldsymbol{a}_{\mathbf{1}} \\
n s / h\end{array}$ \\
\hline R01 & Cs & 2 & 0.715 & 0.106 \\
\hline R02 & Cs & 2 & 0.525 & 0.080 \\
\hline R03 & Cs & 2 & 0.719 & 0.102 \\
\hline R04 & Cs & 2 & 0.654 & 0.092 \\
\hline R05 & Cs & 2 & 0.627 & 0.083 \\
\hline R06 & Cs & 2 & 0.805 & 0.152 \\
\hline R07 & Cs & 2 & 0.717 & 0.100 \\
\hline R08 & Cs & 2 & 0.521 & 0.077 \\
\hline R09 & Cs & 2 & 0.438 & 0.073 \\
\hline $\mathrm{R} 10$ & Cs & 2 & 0.723 & 0.113 \\
\hline $\mathrm{R} 11$ & Cs & 2 & 0.414 & 0.055 \\
\hline $\mathrm{R} 12$ & Cs & 2 & 0.579 & 0.084 \\
\hline $\mathrm{R} 13$ & Cs & 2 & 0.691 & 0.109 \\
\hline $\mathrm{R} 14$ & Cs & 2 & 0.830 & 0.143 \\
\hline $\mathrm{R} 15$ & Cs & 2 & 0.699 & 0.101 \\
\hline $\mathrm{R} 16$ & Cs & 2 & 0.539 & 0.080 \\
\hline R17 & Cs & 2 & 0.449 & 0.065 \\
\hline R18 & Cs & 2 & 0.384 & 0.059 \\
\hline R19 & Cs & 2 & 0.674 & 0.083 \\
\hline $\mathrm{R} 20$ & Cs & 2 & 0.537 & 0.076 \\
\hline $\mathrm{R} 21$ & Cs & 2 & 0.412 & 0.061 \\
\hline $\mathrm{R} 22$ & Cs & 2 & 0.571 & 0.081 \\
\hline $\mathrm{R} 23$ & Cs & 2 & 0.651 & 0.104 \\
\hline $\mathrm{R} 24$ & Cs & 2 & 0.644 & 0.092 \\
\hline & Ave. & & 0.605 & 0.090 \\
\hline
\end{tabular}

Table 3 Optimal algorithm dimension and the STD for $a_{0}, a_{1}$ and $a_{2}$ of BDS

\begin{tabular}{|c|c|c|c|c|}
\hline \multirow{2}{*}{ Sat. } & \multirow{2}{*}{ Dim. } & \multicolumn{3}{|c|}{$12 \mathrm{~h}$ prediction STD } \\
\hline & & $\begin{array}{l}a_{0} \\
n s\end{array}$ & $\begin{array}{c}\boldsymbol{a}_{\mathbf{1}} \\
n s / h\end{array}$ & $\begin{array}{c}\boldsymbol{a}_{\mathbf{2}} \\
n s / h^{2}\end{array}$ \\
\hline $\mathrm{C} 01$ & 3 & 1.319 & 0.183 & 0.012 \\
\hline C02 & 3 & 1.146 & 0.145 & 0.010 \\
\hline C03 & 3 & 1.597 & 0.085 & 0.017 \\
\hline $\mathrm{C} 04$ & 3 & 1.455 & 0.153 & 0.022 \\
\hline $\mathrm{C} 05$ & 3 & 1.524 & 0.253 & 0.015 \\
\hline $\mathrm{C} 06$ & 3 & 5.362 & 0.761 & 0.062 \\
\hline $\mathrm{C} 07$ & 3 & 1.803 & 0.092 & 0.023 \\
\hline $\mathrm{C} 08$ & 3 & 1.823 & 0.172 & 0.017 \\
\hline $\mathrm{C} 09$ & 3 & 1.218 & 0.125 & 0.013 \\
\hline $\mathrm{C} 10$ & 3 & 1.633 & 0.123 & 0.019 \\
\hline $\mathrm{C} 11$ & 3 & 1.008 & 0.078 & 0.005 \\
\hline $\mathrm{C} 12$ & 3 & 0.835 & 0.067 & 0.005 \\
\hline C13 / C15 & 3 & 1.092 & 0.126 & 0.022 \\
\hline $\mathrm{C} 14$ & 3 & 0.686 & 0.055 & 0.004 \\
\hline \multicolumn{2}{|c|}{ Ave. } & 1.607 & 0.172 & 0.017 \\
\hline
\end{tabular}


Table 4 Optimal algorithm dimension and the STD for $a_{0}, a_{1}$ and $a_{2}$ of Galileo

\begin{tabular}{|c|c|c|c|c|c|}
\hline \multirow{2}{*}{ Sat. } & \multirow{2}{*}{ Osc. } & \multirow{2}{*}{ Dim. } & \multicolumn{3}{|c|}{$12 \mathrm{~h}$ prediction STD } \\
\hline & & & $\begin{array}{l}a_{0} \\
n s\end{array}$ & $\begin{array}{c}\boldsymbol{a}_{\mathbf{1}} \\
n s / h\end{array}$ & $\begin{array}{c}\boldsymbol{a}_{\mathbf{2}} \\
n s / h^{2}\end{array}$ \\
\hline E01 & Нy & 3 & 0.351 & 0.0293 & 0.002 \\
\hline E02 & Hy & 3 & 0.319 & 0.0255 & 0.002 \\
\hline E08 & Hy & 3 & 0.784 & 0.0679 & 0.004 \\
\hline E09 & Hy & 3 & 0.861 & 0.0804 & 0.006 \\
\hline E11 & $\mathrm{Rb}$ & 3 & 0.744 & 0.0676 & 0.004 \\
\hline $\mathrm{E} 12$ & Hy & 3 & 0.857 & 0.0856 & 0.006 \\
\hline E14 & $\mathrm{Hy}$ & 3 & 1.086 & 0.1188 & 0.009 \\
\hline $\mathrm{E} 18$ & Hy & 3 & 1.694 & 0.1633 & 0.012 \\
\hline E19 & Hy & 3 & 0.972 & 0.0918 & 0.005 \\
\hline $\mathrm{E} 22$ & $\mathrm{Rb}$ & 3 & 0.987 & 0.0949 & 0.006 \\
\hline $\mathrm{E} 24$ & Hy & 3 & 2.178 & 0.2096 & 0.014 \\
\hline $\mathrm{E} 26$ & Hy & 3 & 0.805 & 0.0743 & 0.005 \\
\hline \multirow[t]{2}{*}{ E30 } & Hy & 3 & 0.859 & 0.0797 & 0.005 \\
\hline & Ave. & & 0.961 & 0.091 & 0.006 \\
\hline
\end{tabular}

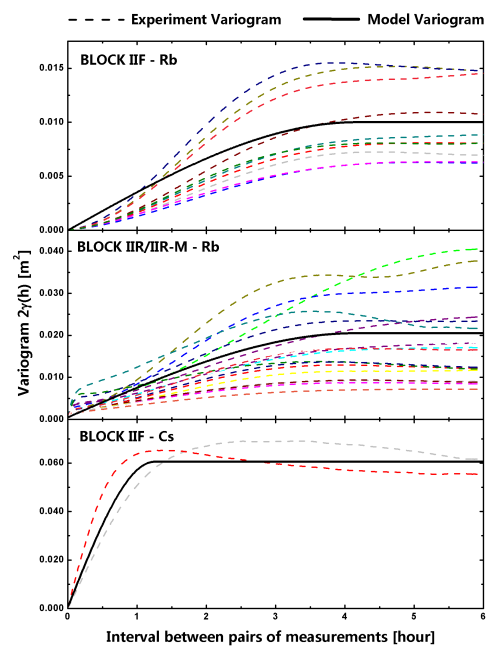

Fig. 1 Variogram from satellite clock fitting residual of model (10) for GPS

the performance of Galileo satellite clock random error $r$ is between that of GPS BLOCK IIF and BLOCK IIR satellite equipped with rubidium oscillator. The comparison between GLONASS and BDS suggests that BDS performs slightly better in terms of $r$ with a longer correlation time. Moreover, from Tab. 3 and Fig. 3 , the oscillator anomaly for C06 can be recognized. 


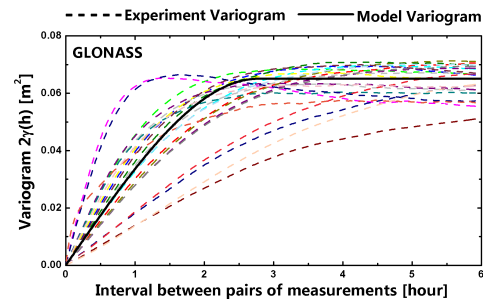

Fig. 2 Variogram from satellite clock fitting residual of model (10) for GLONASS

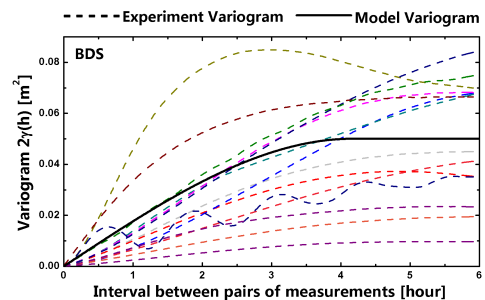

Fig. 3 Variogram from satellite clock fitting residual of model (10) for BDS

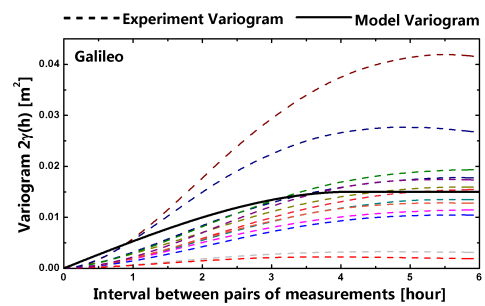

Fig. 4 Variogram from satellite clock fitting residual of model (10) for GALILEO

From the above analysis, a spherical model is selected for the parametric variogram model which is summarized with Eq. (17) and Tab. 5:

$$
\gamma(h)= \begin{cases}0 & , \quad h=0 \\ c_{0}+c_{s}\left\{(3 / 2)\left(h / a_{s}\right)-(1 / 2)\left(h / a_{s}\right)^{3}\right\} & , \quad 0<h<a_{s} \\ c_{0}+c_{s} & , \quad h \geq a_{s}\end{cases}
$$

As an illustration, the model variogram based on Eq. (17) and Tab. 5 is also plotted in the corresponding figure with solid black line. For more details concerning variogram, we refer to [Cressie (1993)] and [Shi et al. (2012)].

\subsection{Discussion}

Before the solution of the GNSS satellite clock estimation based on Eq. (1) and Eq. (11), there are actually more issues left to be solved, i.e., the rank deficiency 
Table 5 Values of $c_{0}, c_{s}$ and $a_{s}$ for $G, R, C, E$

\begin{tabular}{clccc}
\hline & Sys. & $\boldsymbol{c}_{\mathbf{0}}$ & $\boldsymbol{c}_{\boldsymbol{s}}$ & $\boldsymbol{a}_{\boldsymbol{s}}$ \\
\hline \multirow{4}{*}{ GPS } & IIF - Rb & 0 & 0.005 & 15000 \\
& IIR/IIR-M - Rb & 0.0002 & 0.01 & 15000 \\
& IIF - Cs & 0.0002 & 0.03 & 4500 \\
& GLONASS & 0 & 0.032 & 10000 \\
BDS & 0 & 0.023 & 15000 \\
& Galileo & 0 & 0.0075 & 15000 \\
\hline
\end{tabular}

raised from the linear dependency of clock and code bias terms. To reduce this datum deficiency problem, condition Eq. (18) is introduced in this study to make model (1) and (11) uniquely solvable.

$$
0=u \cdot a_{i}^{s y s}
$$

with $\boldsymbol{u}=\left(\begin{array}{llll}1 & 1 & \cdots & 1\end{array}\right)^{T}$. The subscript $i \in\left(\begin{array}{lll}0 & 1 & 2\end{array}\right)$ and sys $\in\left(\begin{array}{lll}G R C E & R \text {, thus } \boldsymbol{a}_{\boldsymbol{i}}^{\text {sys }}\end{array}\right.$ stands for the vector of $a_{i}$ of different satellites for sys. As the rank deficiency problem is beyond the scope of this contribution, for more details we refer to the work of [Blaha (1982)] and [Gu et al. (2013)]. In the former work, the generally concept of free network solution is presented, while the application of this concept in the GNSS data processing is discussed in the later one.

Moreover, as indicated by Eq. (11) to Eq. (13), the terms $\left(a_{0} a_{1} a_{2}\right)$ are assumed as constant, while, the clock bias $a_{0}$ is indeed a constant by definition, the frequency bias and drift parameters are not strictly constant, but subject to random fluctuations. These variation effects on the frequency bias and drift are analyzed based on the Allan and Hadamard variance respectively. Once the Allan and Hadamard variance obtained, a different oscillator noise model may derived as presented by [Stein (1989)] and [Huang et al. (2013B)].

However in this contribution, we chose to follow the strategy of [Hauschild and Montenbruck (2009), Hauschild (2010)], and treat the frequency bias and drift as constant, and compensate the deviations of the system model from the truth with a process noise parameter $r$ since that: this oscillator noise model is built for real time clock estimation with a regional network, thus the continuous data length of each satellite is typically less than 12 hours, in which case, the terms $\left(a_{0} a_{1} a_{2}\right)$ can be safely regarded as constant as demonstrated in the following sections.

\section{Experiment}

\subsection{Software Development and Data Collection}

To cope with the upcoming or available new and modernized GNSS signals, especially, the multi-frequency data processing, real-time applications, as well as the GNSS/INS integrated navigation, we launched the FUSING (FUSing IN Gnss) project. At present, the FUSING software is capable for the precise orbit determination (POD) of GNSS, high-frequency satellite clock estimation, ionosphere and troposphere modeling as well as multi-frequency precise positioning. In this study, we have developed a new module with the oscillator noise model described 


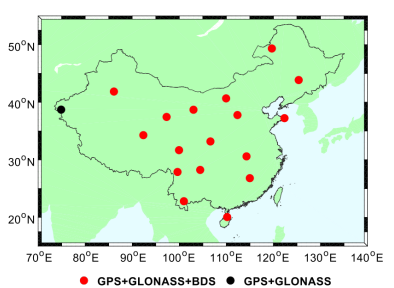

Fig. 5 Distribution of the 18 regional GNSS stations; points in red represent stations capable of tracking $G, R, C$ and one station capable of tracking $G, R$ in black

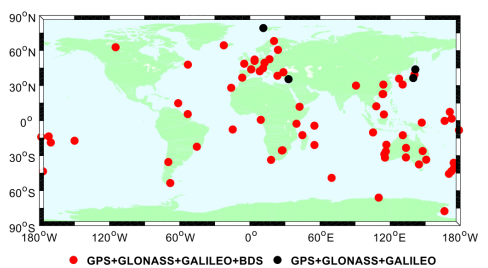

Fig. 6 Distribution of the 80 global GNSS stations; points in red represent stations capable of tracking $G, R, C, E$ and four stations capable of tracking $G, R, E$ in black

in section 3 into the FUSING software. In the following demonstration, the new module is assessed with both regional and global networks for simulated real-time multi-GNSS $G, R, C, E$ satellite clock estimation. And for comparison purpose, the traditional approach based on white noise model is also presented.

Shown in Fig. 5 and Fig. 6 are the distribution of 18 and 80 stations for a regional and global network, respectively. For the regional experiment, the data is collected by the crustal movement observation network of China (CMONOC) from DOY 153 to 182,2016 with an interval of $30 \mathrm{~s}$. It is a pity that the Galileo is not valid in the regional experiment, while, for the global experiment, the data is collected by the multi-GNSS experiment campaign (MGEX) from DOY 001 to 030 of 2017 with an interval of $30 \mathrm{~s}$. Concerning the processing strategy of these experiments, please refer to Tab. 6. As indicated in Tab. 6, the satellite clock products estimated with no less than three stations are all included in the statistical analysis, i.e., the result is also affected by the convergence. In addition, we have ignored the effect of GLONASS inter-frequency code bias in this study.

\subsection{Regional Experiment}

\subsubsection{Satellite discontinuity}

To begin with the evaluation of the regional experiment, Tab. 7 illustrates the typical satellite tracking continuity averaged against the corresponding orbit period for a simulated network evenly distributed in China. Though the Galileo is not 
Table 6 Details of the satellite clock estimation strategy

\begin{tabular}{cl}
\hline Item & Strategy \\
\hline Observable & Ionosphere free combination \\
Solution & Day by day with $G, R, C, E$ in one square root information filter \\
\hline $\begin{array}{c}\text { Station coordinate } \\
\text { Satellite orbit }\end{array}$ & Fixed with static PPP solution averaged over one week using PANDA \\
PCO/PCV & Fixed with GFZ final multi-system orbit products \\
Tides & Corrected \\
Phase windup & Corrected \\
Receiver clocks / ISBs & One clock for each system as white noise parameter, and no ISBs are involved \\
Satellite clocks $\quad$ ON & Estimated as Oscillator Noise parameters of model (11) for each satellite, and $a_{1}$ and $a_{2}$ are reset day by day \\
Troposphere & Estimated as White Noise parameter for each satellite \\
Ambiguity & Prior model with remaining estimated as a random walk process \\
Elevation angle cutoff & Constant for each continuous arc \\
Weighting & 8 dm for pseudo-range and 8 mm for phase, low elevation observable and outliers are down-weighted \\
\hline Quality control & Satellite clocks are generated only for those with at least three stations tracked simultaneously \\
Reference & GFZ final multi-system clock products \\
\hline
\end{tabular}

tracked by CMONOC up to now, the result is still presented in Tab . 7. It is concluded that the gap between the adjacent arcs is $6 \sim 9$ hours for the MEO satellites over China. Moreover, it should be noted that the actual tracking arc is subject to the status of satellites as well as stations. As an example, Fig. 7 presents the visibility of satellite G01 from different stations for DOY 153, 2016 with a cutoff angle of $7^{\circ}$ from our experiment, while, the tracking arc for all satellites of this day is plotted in Fig. 8.

Table 7 The typical satellite tracking continuity averaged against the corresponding orbit period for a network evenly distributed in China

\begin{tabular}{cccc}
\hline Sys. & Period [Day] & Tracked [h] & Gap [h] \\
\hline GPS & 1 & 9.19 & 6.08 \\
GLONASS & 8 & 6.51 & 4.74 \\
BDS IGSO & 1 & 20.03 & 3.30 \\
Galileo & 7 & 6.13 & 7.70 \\
& 10 & 6.66 & 9.01 \\
\hline
\end{tabular}

\subsubsection{Clock evaluation}

Differenced with the GFZ final clock products, Fig. 9 gives the statistics of the regional satellite clock solution averaged over each constellation $G, R, C$ from DOY 153 to 182,2016 . Since there may be more than one arc for each satellite during each day in the regional experiment, the STD in Fig. 9 is the mean value averaged over all STDs for different arcs which can be expressed as

$$
S T D=\frac{\sum_{i=1}^{m} S T D_{i}}{m}
$$




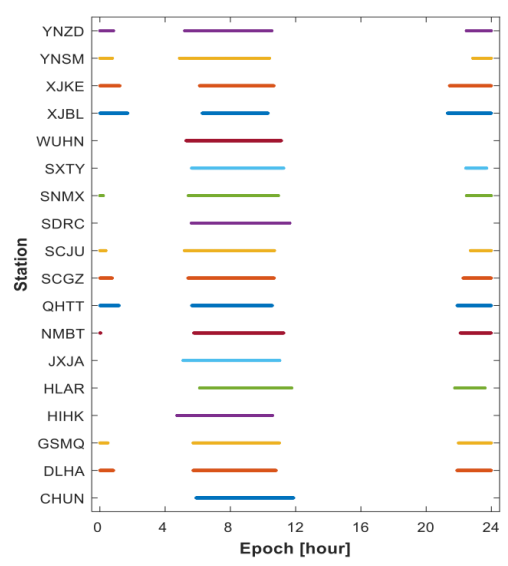

Fig. 7 Visibility of satellite G01 from different stations for DOY 153, 2016 with a cutoff angle of $7^{\circ}$

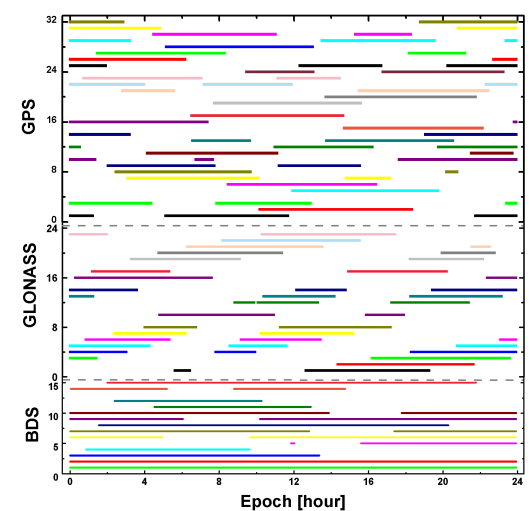

Fig. 8 The typical satellite tracking arc of GPS, GLONASS and BDS with CMONOC for DOY 153,2016

where $S T D_{i}=\sqrt{\frac{\sum_{j=1}^{n}\left(d t_{j}-\bar{d} t_{i}\right)}{n-1}}$ and $\bar{d} t_{i}=\frac{\sum_{j=1}^{n} d t_{j}}{n}$ are the STD and mean clock bias for arc $i$, respectively. The RMS is derived over all the samples, i.e., regardless the arcs of the satellite for each day. Note that both the samples before and after convergence are included in the STD and RMS calculation, i.e., the convergence periods after the data discontinuity are also included in the evaluation of the clock solutions based on oscillator noise model and white noise model. It is obvious that when only regional stations involved, the performance of the satellite clock estimates can be improved significantly constrained with the oscillator noise model presented in section 3.1. Compared with the results based on white noise model, 


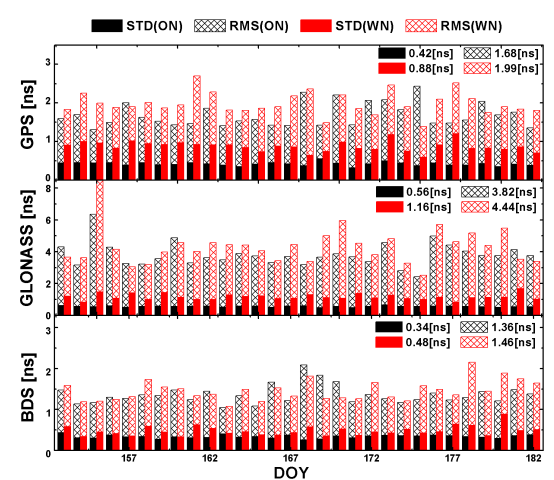

Fig. 9 Statistics of the satellite clock estimation of GPS, GLONASS and BDS with CMONOC from DOY 153 to 182,2016

the improvement is $44.4 \%$ and $12.1 \%$ on average for STD and RMS, respectively. Furthermore, the comparison among the $G, R, C$ solutions suggests that the oscillator noise model is less efficient for the BDS solution. This is mainly due to the fact that the constellation of BDS mainly consists with the GEO and IGSO satellites, which suffer less data discontinuity. And for the same reason, the BDS satellite clock presents the best results with a STD of $0.34 n$ s and RMS of 1.36ns.

Furthermore, Fig. 10 illustrates the $95 \%$ and $68 \%$ confidence level convergence series of a 6-hour pass during the initialization averaged over all the samples of the regional experiment. Together with Fig. 9, it is concluded that the improvement in the statistics, i.e., STD and RMS, are mainly attributed to the efficiency of the oscillator noise model during the first few hours for $G, R$. And not surprising, the improvement in convergence of $\mathrm{BDS}$ is not as evident as that of $G, R$. As presented in Tab. 8 , the statistics based on samples after convergence, i.e., 3 hours later since the start of each satellite tracking arc, suggested that the precision of the solutions with different models are roughly the same after convergence. While, though the improvements after convergence are rather limited, it is still expected that the solution with oscillator noise model is more resistant to the gross error, especially during the end of each arc for satellite with a low elevation angle. This statement is confirmed with the clock difference series of G22, R12 and C12, respectively, for DOY 159, 2016 in Fig. 11.

Table 8 Statistics of the satellite clock estimation of GPS, GLONASS and BDS based on samples after convergence, i.e., 3 hours later since the start of each satellite tracking arc with from DOY 153 to 182

\begin{tabular}{ccccc}
\hline Sys. & & GPS & GLONASS & BDS \\
\hline \multirow{2}{*}{ STD [ns] } & OM & 0.19 & 0.22 & 0.20 \\
& WN & 0.19 & 0.31 & 0.20 \\
\multirow{2}{*}{ RMS [ns] } & OM & 1.36 & 3.33 & 1.23 \\
& WN & 1.35 & 3.54 & 1.29 \\
\hline
\end{tabular}




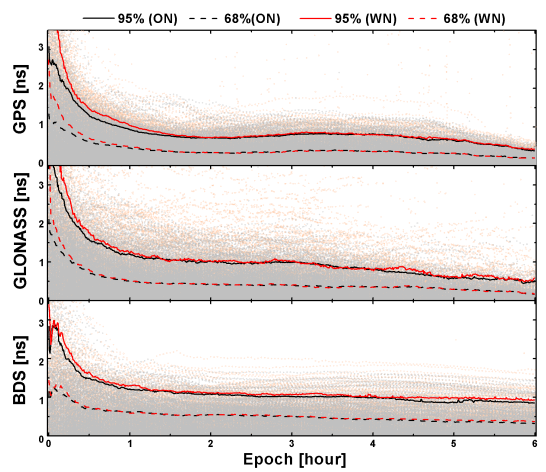

Fig. 10 The $95 \%$ and $68 \%$ confidence level convergence series of a 6 -hour pass averaged over DOY 153 to 182, 2016 for GPS, GLONASS and BDS with CMONOC. While, the background scatter are the satellite clock convergence series for each individual arc for $\mathbf{O N}$ (gray) and WN (orange)
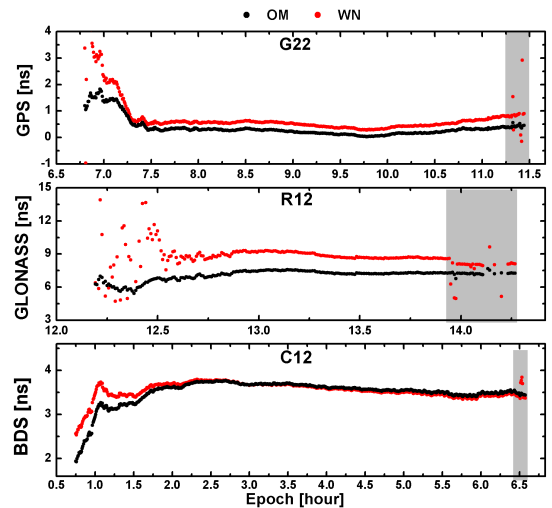

Fig. 11 Clock difference series of G22, R12 and C12, respectively, for DOY 159, 2016. The black dot denotes the solution with oscillator noise model, while, the red dot denotes the solution with white noise model

\subsection{Global Experiment}

Though this contribution aims at the satellite clock estimation with regional stations constrained with oscillator noise model, a global experiment was also carried out for comparison purpose.

In a similar manner of the regional experiment, presented in Fig. 12 are the STD and RMS averaged over DOY 001 to 030, 2017 for $G, R, C, E$ with the global network. Generally speaking, the precision is at the same level regardless the clock model involved as demonstrated by Fig. 12. Apparent improvement can only be found in Galileo satellites with the STD decreased from $0.28 n s$ to $0.19 n s$. A detailed analysis reveals that even with the stations in Fig. 6, the satellites $E 14$ and E18 still suffer significant data discontinuity, i.e., $7.4 h /$ day for our experimental 


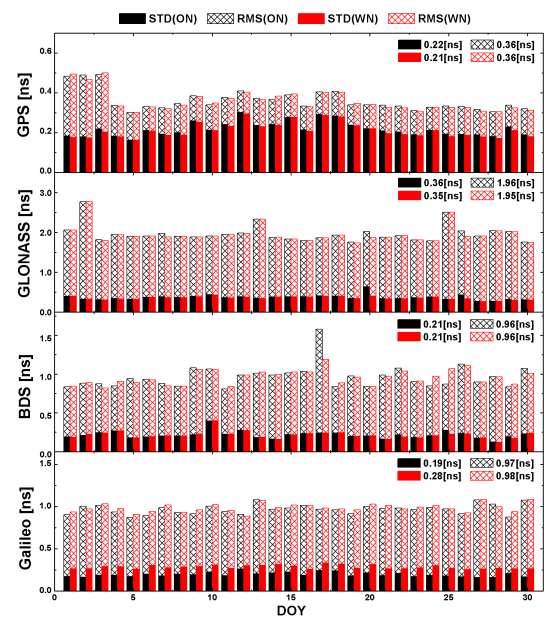

Fig. 12 Statistics of the satellite clock estimation of GPS, GLONASS, BDS and Galileo with MGEX from DOY 001 to 030, 2017

period. While the STD and RMS are improved to $0.19 n s$ and $0.96 n s$, respectively, once we exclude $E 14$ and $E 18$ in the statistics. Though the root cause of these abnormal remains unknown, it is most likely due to the orbit recovery of these two satellites: E14 and E18 [Männel (2017)]. This result again confirms that the clock estimation in the existence of data discontinuity is expected to be benefited from the oscillator noise model promoted in this study.

Overall, the STD for the satellite clock with global network is about $0.2 n s$ for $G, C, E$ based on the whole daily session. While after convergence, the STD is improved to about $0.05 \mathrm{~ns}$ as demonstrated in the previous studies of involving authors in [Gong et al. (2017)]. Compared with the results of $G, C, E$, the STD and RMS of GLONASS are much worse with a value of $0.36 \mathrm{~ns}$ and $1.96 \mathrm{~ns}$, respectively. This is mainly due to the fact that the GLONASS inter-frequency code bias has not been taken into consideration in this study, while they have been estimated in the GFZ products. In addition, since the clock estimates depend on the type of observations and no official differential code biases (DCB) for Galileo are available, the solutions of this study and GFZ based on different stations may result in systemic bias [Gong et al. (2017)].

Furthermore, Fig. 13 presents the daily repeatability of frequency bias and frequency drift, i.e., $a_{1}$ and $a_{2}$ for each satellite of $G, R, C, E$ with MGEX averaged over DOY 001 to 030,2017 . While the statistics of $a_{0}$ is not presented, the repeatability of $a_{0}$ is subjected to the daily boundary of the initial clock involved. It is concluded from Fig. 13, that the oscillator stability of Galileo is comparable with that of GPS, which is about $0.05 n s / h$ for $a_{1}$ and $0.002 n s / h^{2}$ for $a_{2}$, while the STD of BDS is extremely larger than that of $G, R, C$. Besides the quality of the BDS oscillator itself, another possible reason is that the clock estimates may absorb the daily boundary of the BDS orbit. Additionally, it is noted that the frequency bias of Galileo is much larger than that of $G, R, C$. 


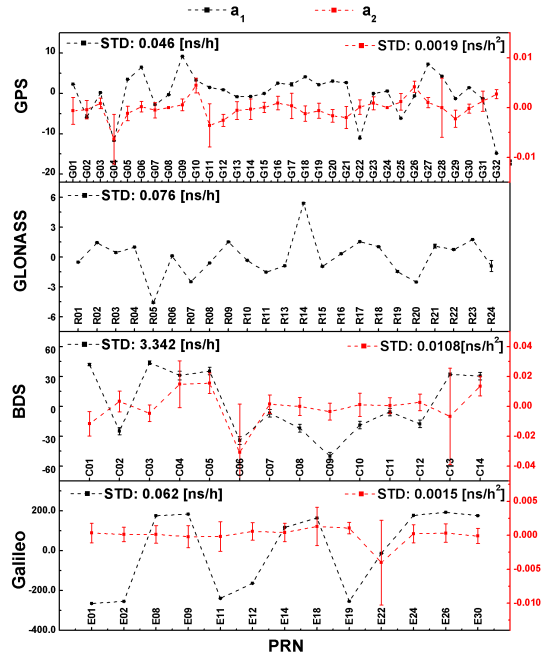

Fig. 13 Daily repeatability of the oscillator noise model parameters $a_{1}$ (in black) and $a_{2}$ (in red) for GPS, GLONASS, BDS and Galileo with MGEX. The STD presented in this figure is the averaged value of the statistics for each satellite from DOY 001 to 030,2017 . Note that the satellite E22 has been removed in the statistic of Galileo as outlier

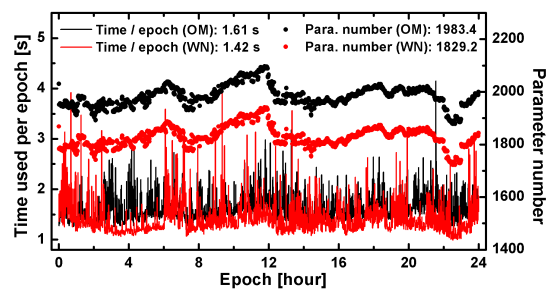

Fig. 14 Execution time per epoch and parameter number for DOY 001, 2017. The black line and dot denote the results of clock solution with oscillator noise model, while, the red line and dot denote the results of clock solution with white noise model

Besides the precision, the computational efficiency presents another key factor in satellite clock solution. Thus, Fig. 14 illustrates the processing efficiency and the parameter number of the satellite clock estimation with oscillator noise model and white noise model denoted in black and red, respectively. The analysis is conducted on a Centos 7 personal laptop with an Intel Core-3.3 GHz processor and 16 GB memory. Overall, more data processing effort is required with the new model due to the extra 154.2 parameters on average. Whereas, this extra time, i.e., $0.19 \mathrm{~s}$ per epoch, is negligible and the total execute time per epoch, i.e., $1.61 \mathrm{~s}$, can fully satisfy the requirement of real-time precise satellite clock estimation. The processing efficiency here is comparable with our previous work for high efficient undifferenced GNSS data processing [Gong et al. (2017)]. 


\section{Conclusion}

The purpose of this contribution is to explore the potential of the oscillator noise model to constrain the real-time satellite clock estimation in the existence of the data discontinuity, i.e., regional network solution. Thus, the temporal behavior of the satellite oscillator was studied for GPS, GLONASS, BDS and Galileo based on the GFZ final multi-GNSS clock product from May. 3rd, 2015 to Feb. 25th, 2017. Different oscillator noise model were promoted and assigned to different satellites according to its oscillator type as well as the block type. In this oscillator noise models, the clock bias, frequency bias and frequency drift were estimated as constant, and the deviations of the model from the truth are further compensated with a random walk parameter.

By employing this model into the simulated real-time multi-GNSS clock solution, estimates of GPS, GLONASS and BDS satellite clock were derived with the CMONOC network with 18 stations evenly distributed over China from DOY 153 to 182,2016 . The comparison between the solution constrained with the oscillator noise model and the traditional solution with white noise demonstrates the efficiency of the proposed model with an improvement of $44.4 \%$ and $12.1 \%$ on average for STD and RMS, respectively. Furthermore, a global experiment was performed based on the MGEX network with 80 stations distributed worldwide from DOY 001 to 030, 2017. Though the precision of the clock estimates are comparable for these solutions with different models for GPS, GLONASS and BDS, the STD of Galileo clock improves from $0.28 n s$ to $0.19 n s$ due to that, the satellites E14 and E18 still suffer significant data discontinuity, i.e., 7.4h/day.

It should be mentioned, although rarely happened, that the satellite clock slip of BDS is detected from our experiment, and special attention should be given to this phenomenon when employing the oscillator noise model in the satellite clock estimation. Moreover, though the oscillator noise model seems redundant once the satellites are being tracked continuously, it still deserves to be studied: firstly, there are cases that the clock estimates should be predicted due to the connection problem of the real-time PPP users; secondly, the stability and smoothness of clock estimates are essential in the GNSS based timing and synchronization; and finally, with the development of the GNSS oscillators in the future, more precise constrains can be applied, which has the potential to further improve the performance.

\section{Acknowledgements}

This study is sponsored by the National Key Research and Development Plan (No. 2016YFB0501802). The authors thank the anonymous reviewers for their valuable comments. Thanks also go to IGS for data provision.

\section{References}

[Allan (1987)] Allan DW (1987) Time and frequency(time-domain) characterization, estimation, and prediction of precision clocks and oscillators. IEEE Transactions on Ultrasonics, 34(6), 647-654. http://doi.org/10.1109/t-uffc.1987.26997

[Bierman (1975)] Bierman GJ (1975) The treatment of bias in the square-root information filter/smoother. Journal of Optimization Theory and Applications, 16(1-2), 165-178. http://doi.org/10.1007/BF00935630 
[Bisnath and Gao (2008)] Bisnath S, Gao Y (2008) Current State of Precise Point Positioning and Future Prospects and Limitations. In Observing our Changing Earth (Vol. 133, pp. 615?623). Berlin, Heidelberg: Springer Berlin Heidelberg. http://doi.org/10.1007/978-3-54085426-5_71

[Blaha (1982)] Blaha G (1982) Free networks: minimum norm solution as obtained by the inner adjustment constraint method. Bulletin Géodésique, 56(3), 209-219. http://doi.org/10.1007/BF02525582

[Bock et al. (2009A)] Bock H, Dach R, Jaeggi A, Beutler G (2009A) High-rate GPS clock corrections from CODE: support of $1 \mathrm{~Hz}$ applications. Journal of Geodesy, 83(11), 10831094. http://doi.org/10.1007/s00190-009-0326-1

[Bock et al. (2009B)] Bock H, Dach R, Yoon Y, Montenbruck O (2009B) GPS clock correc-

tion estimation for near real-time orbit determination applications. Aerospace Science and Technology, 13(7), 415-422. http://doi.org/10.1016/j.ast.2009.08.003

[Cressie (1993)] Cressie N (1993) Statistics for spatial data. John Wiley \& Sons Inc, pp. 928, 1993

[Cressie and Hawkins (1980)] Cressie N, Hawkins DM (1980) Robust estimation of the variogram: I. Mathematical Geology. 12(2), pp. 115-125, doi:10.1007/BF01035243, 1980

[Ge et al. (2011)] Ge M, Chen J, Douša J, Gendt G, Wickert J (2011) A computationally efficient approach for estimating high-rate satellite clock corrections in realtime. GPS Solutions, 16(1), 9-17. http://doi.org/10.1007/s10291-011-0206-z

[Gong et al. (2017)] Gong X, Gu S, Lou Y, Zheng F, Ge M, Liu J (2017) An efficient solution of real-time data processing for multi-GNSS network. Journal of Geodesy 16:1-13. doi: $10.1007 / \mathrm{s} 00190-017-1095-\mathrm{x}$

[Gu et al. (2013)] Gu S, Shi C, Lou Y, Feng Y, Ge M (2013) Generalized-positioning for mixedfrequency of mixed-GNSS and its preliminary applications. In: Proceedings on China satellite navigation conference (CSNC), pp 399?428. http://doi.org/10.1007/978-3-642-37404-3_35

[Hauschild (2010)] Hauschild A (2010) Precise GNSS clock-estimation for real-time navigation and Precise Point Positioning. Technical University of Munich, Munich.

[Hauschild and Montenbruck (2009)] Hauschild A, Montenbruck O (2009) Kalman-filterbased GPS clock estimation for near real-time positioning. GPS Solutions, 13(3), 173-182. http://doi.org/10.1007/s10291-008-0110-3

[Huang et al. (2012)] Huang GW, Zhang Q (2012) Real-time estimation of satellite clock offset using adaptively robust Kalman filter with classified adaptive factors. GPS Solutions, 16(4), 531-539. http://doi.org/10.1007/s10291-012-0254-z

[Huang et al. (2013A)] Huang GW, Zhang Q, Li H, Fu W (2013A) Quality variation of GPS satellite clocks on-orbit using IGS clock products. Advances in Space Research, 51(6), 978987. http://doi.org/10.1016/j.asr.2012.09.041

[Huang et al. (2013B)] Huang GW, Zhang Q, Xu GC (2013B) Real-time clock offset prediction with an improved model. GPS Solutions, 18(1), 95-104. http://doi.org/10.1007/s10291-0130313-0

[Hutsell (1995)] Hutsell Capt Steven T(1995) Relating the Hadamard Variance to MCS Kalman Filter Clock Estimation, Proceedings of the 27th Annual Precise Time and Time Interval Systems and Applications Meeting, San Diego, California, November 1995, 291-302.

[Kouba and Héroux. (2001)] Kouba J, Héroux P (2001) Precise point positioning using IGS orbit and clock products. GPS Solutions, 5(2), 12-28.

[Laurichesse et al. (2013)] Laurichesse D, Cerri L, Berthias JP, Mercier F (2013) Real time precise GPS constellation and clocks estimation by means of a Kalman filter. Presented at the 26th International Technical Meeting of the Satellite Division of the Institute of Navigation, ION GNSS 2013

[Lindsey (1976)] Lindsey W (1976) Theory of Oscillator Instability Based Upon Structure Functions. Proceedings of the IEEE, 64(12), 1652-1666. http://doi.org/10.1109/PROC.1976.10408

[Liu et al. (2017)] Liu Y, Ye S, Song W, Lou Y, Gu S (2017) Rapid PPP ambiguity resolution using GPS+GLONASS observations. Journal of Geodesy, 1-15. http://doi.org/10.1007/s00190-016-0975-9

[Lou et al. (2015)] Lou Y, Zheng F, Gu S, Wang C, Guo H, Feng Y (2015) Multi-GNSS precise point positioning with raw single-frequency and dual-frequency measurement models. GPS Solutions, 20(4), 849-862. http://doi.org/10.1007/s10291-015-0495-8

[Männel (2017)] ZHA15 Männel B (2017) Reprocessing the Elliptical Orbiting Galileo Satellites E14 and E18: Preliminary Results. Presented at the EGU General Assembly 2017. 
[Montenbruck et al. (2012)] Montenbruck O, Hauschild A, Steigenberger P, Hugentobler U, Teunissen PJG, Nakamura S (2012) Initial assessment of the COMPASS/BeiDou-2 regional navigation satellite system. GPS Solutions, 17(2), 211-222. http://doi.org/10.1007/s10291012-0272-x

[Petit and Thomas (1996)] Petit G, Thomas C (1996) GPS frequency transfer using carrier phase measurements. Presented at the 1996 IEEE International Frequency Control Symposium, 1151-1158, IEEE. http://doi.org/10.1109/FREQ.1996.560307.

[Senior et al. (2008)] Senior KL, Ray JR, Beard RL (2008) Characterization of periodic variations in the GPS satellite clocks. GPS Solutions, 12(3), 211-225. http://doi.org/10.1007/s10291-008-0089-9.

[Shi et al. (2012)] Shi C, Gu S, Lou Y, Ge M (2012) An improved approach to model ionospheric delays for single-frequency precise point positioning. Adv Space Res 49(12):16981708. doi:10.1016/j.asr.2012.03.016

[Shi et al. (2013)] Shi C, Lou Y, Song W, Gu S, Geng C, Yi, W, Liu Y (2013) A Wide Area Real-Time Differential GPS Prototype System in China and Result Analysis. Survey Review, 43(322), 351-360. http://doi.org/10.1179/003962611X13055561708623.

[Shi et al. (2017)] Shi C, Zheng F, Lou Y, Gu S, Zhang W, Dai X, Li X, Guo H, Gong X (2017) National BDS Augmentation Service System (NBASS) of China: Progress and Assessment. Remote Sensing, 9(8). http://doi.org/10.3390/rs9080837

[Shmaliy et al. (1999)] Shmaliy YS, Marienko AV, Savchuk AV (1999) GPS-based optimal Kalman estimation of time error, frequency offset, and aging. Presented at the 31st Annual Precise Time and Time Interval Meeting, 1999, pp.431?440

[Steigenberger et al. (2013)] Steigenberger P, Hugentobler U, Hauschild A, Montenbruck O (2013) Orbit and clock analysis of Compass GEO and IGSO satellites. Journal of Geodesy, 87(6), 515-525. http://doi.org/10.1007/s00190-013-0625-4

[Stein (1989)] Stein SR (1989) Kalman filter analysis of precision clocks with real-time parameter estimation. Proceedings of the 43rd Annual Symposium on Frequency Control, 1989, pp. 232- 236.

[Swift and Hermann (1988)] Swift ER, Hermann BR (1988) Orbit period frequency variations in the GPS satellite clocks. In: Proceedings of the 20th annual precise time and time interval (PTTI) applications and planning meeting, Vienna, VA (USA), Nov 29?Dec 1, pp. 87-100

[Wang et al. (2017)] Wang B, Lou Y, Liu J, Zhao Q, Su X (2017) Analysis of BDS satellite clocks in orbit. GPS Solutions, 20(4), 783-794. http://doi.org/10.1007/s10291-015-0488-7

[Weber et al. (2007)] Weber G, Mervart L, Lukes Z, Rocken C, Dousa J (2007) Real-time clock and orbit corrections for improved point positioning via NTRIP. In: Proceedings of ION-GNSS-2007.

[Wright (2007)] Wright JR (2007) GPS Composite Clock Analysis. Presented at the Frequency Control Symposium, 2007 Joint with the 21st European Frequency and Time Forum. 523528, IEEE International, IEEE. http://doi.org/10.1109/FREQ.2007.4319128

[Wübbena (1988)] Wübbena G (1988) GPS carrier phases and clock modeling. In GPSTechniques Applied to Geodesy and Surveying. 19, 381-392. Berlin/Heidelberg: SpringerVerlag. http://doi.org/10.1007/BFb0011350.

[Zhang et al. (2015)] Zhang W, Lou Y, Gu S, Shi C, Haase JS, Liu J (2015) Joint estimation of GPS/BDS real-time clocks and initial results. GPS Solutions, 20(4), 665-676. http://doi.org/10.1007/s10291-015-0476-y

[Zumberge et al. (1997)] Zumberge JF, Heflin MB, Jefferson DC, Watkins M, Webb FH (1997)

Precise point positioning for the efficient and robust analysis of GPS data from large networks. J Geophys Res. 102(B3),5005-5017. 